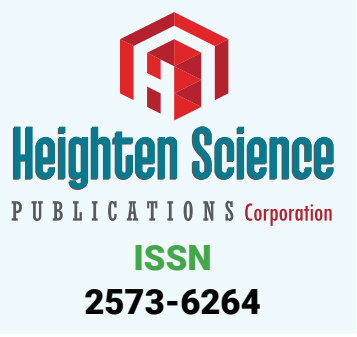

*Address for Correspondence: Camila Fonseca de Oliveira. Center of Research, Education, Innovation and Intervention in Sport, Faculty of Sport, University of Porto, Porto Portugal, Email: fisiocamilafonseca@gmail.com

Submitted: 26 December 2016

Approved: 18 January 2017

Published: 20 January 2017

Copyright: @ 2017 de Oliveira et al. This is an open access article distributed under the Creative Commons Attribution License, which permits unrestricted use, distribution, and reproduction in any medium, provided the original work is properly cited.

Keywords: Coactivation; Electromyography; Falls in elderly; Fatigue; Activities of daily living

\title{
Effects of Fast-Walking on Muscle Activation in Young Adults and Elderly Persons
}

\author{
Camila Fonseca de Oliveira ${ }^{1,2}$, Denise Paschoal Soares ${ }^{1,2}$, \\ Michel Christian Bertani ${ }^{1,2}$, Leandro José Rodrigues \\ Machado ${ }^{1,2}$ and João Paulo Vila-Boas ${ }^{1,2}$ \\ ${ }^{1}$ Center of Research, Education, Innovation and Intervention in Sport, Faculty of Sport, \\ University of Porto, Porto Portugal \\ ${ }^{2}$ Porto Biomechanics Laboratory, University of Porto, Porto, Portugal
}

\section{ABSTRACT}

Coactivation of agonist and antagonist muscles participates in the regulation of joint stiffness and postural instability. Alterations on muscle activity have been revealed as an important falling risk factor. It is unclear the effects, and age-related differences, of a prolonged demanding task on the muscular coactivation levels. We compared muscle activation amplitude and coactivation of the vastus medialis, biceps femoris, tibialis anterior, and gastrocnemius medialis from surface EMG in 16 young adults (age 21-33) and 8 elderly adults (age 66-72) while fast-walking at $70 \%$ of their maximum heart rate. Overall, the elderly demonstrated higher coactivation indexes than the young individuals. Ankle coactivation decreased in the first half of the swing phase, while coactivation at the knee increased in the latter half of the swing phase in our elders. Alterations of muscle activation and coactivation on the knee and ankle were more prominent close to landing and in the swing phase. Our results suggest that these alterations may suggest potential concerns with respect to the risk of falls.

\section{INTRODUCTION}

The aging of the population is a worldwide phenomenon. In 2050, Europe will have an elderly population of $31.9 \%$, i.e. one in each three people will be over the age of 65 [1]. This demographic behavior has led to new challenges in public health given the financial impact caused by the number of hospitalizations of this population, most of them due to consequences of falls. Risk factors of falls in elders are usually related to the unsuccessful interactions between environmental demands and intrinsic factors [2]. Age-related decrements in neuromuscular function, control of balance and strength, are all key factors that when isolated or combined make the elderly more susceptible to falls [2,3]. Since most of falls occur during locomotion, decrements in dynamic postural control and gait performance has been pointed as risk factors for falling [4].

Stable walking kinematics requires generating appropriate motor patterns, in which muscle coactivation plays an important role [4]. In a variety of activities, including walking, elderly use more coactivation than young adults [5]. When walking at a faster pace, coactivation of antagonist leg muscles has shown to be greater in older adults and to have a high association to metabolic rate [6]. Thus, an activity that is more energy consuming, such as fast walking, which implies higher coactivation levels, may compromise gait performance over time in older adults. According to [7], 
prolonged walking on a treadmill induced changes in the lower limb muscle activities, however how the muscle activation patterns changes over time during fast walking were not described. Although a prior study regarding effects of fast-walking on the pace of elders suggested that it was safe to perform fast-speed walking exercises [8]. Nagano and collaborators [9] demonstrated that after a short period of a fast walk older individuals were more susceptible to falls by tripping. Thus, it remains unclear whether fast-walking can be performed without imposing risk to the elderly.

Coactivation of agonist and antagonist muscles participates in the regulation of joint stiffness and postural instability $[5,10]$. Thus, alterations on muscle activity may reflect walking stability. To understand fast-walking effects on gait performance, it is of interest to analyze the neuromuscular alteration over time.

The purpose of this study was to investigate the influence of fast-walking on activation and coactivation of lower limb muscles of young and older adults. We hypothesized that older adults would have greater muscle activation and coactivation than the younger individuals during a gait, and that the levels of muscle activation and coactivation would increase throughout the task.

\section{METHODS}

\section{Participants}

Data of 15 adults $(27 \pm 5$ years; $1.68 \pm 0.12 \mathrm{~m} ; 68.3 \pm 17.3 \mathrm{Kg}$ ) and 8 elderly persons ( $69.6 \pm 3.8$ years; $1.56 \pm 0.1 \mathrm{~m} ; 66.9 \pm 8.2 \mathrm{~kg}$ ) were considered for this study. Adults were recruited from among the students of the University and elderly participants were recruited from the local community to engage in this study. The subjects are described in table 1. The exclusion criteria were any orthopedic, neurological, visual, vestibular or cardiovascular conditions that would not allow the subject to perform all of the proposed activities. All participants gave signed consent to participate in the study. This research was approved by the Local Ethics Committee.

\section{Task and procedures}

All participants completed a familiarization session on an instrumented treadmill (AMTI Inc, MA, USA) walking at their preferred walking speed for five minutes. Elderly men and women wore a safety harness attached to the ceiling. After a familiarization period of 5 minutes, the treadmill speed was increased by $0.5 \mathrm{~km} / \mathrm{h}$ every 30 seconds until everybody reached the speed where they would be at $70 \%$ of their age-predicted maximal heart rate (220 minus age in years). The participants were instructed to walk at this intensity for twenty minutes or until the voluntary exhaustion. Heart rate (HR) was measured continuously during the test with a Polar HR monitor (2010 Polar Electro Oy, FI-90440 Kempele, Finland) to ensure they were walking at the required intensity. Additionally, the rating of perceived exertion scale was obtained throughout the protocol using a modified Borg 10-point scale [11].

Data were recorded for 30 seconds every minute until the end of the protocol. Since not all the participants were able to walk during twenty minutes, five time points equally distributed were used to analyze the data over time. The first and the last thirty seconds of the recorded data, the second time point was the 30s recorded at the middle point between the $1^{\text {st }}$ and the last, and the second and fourth time point from the middle time between the first and the third time point, and the third and last time point, respectively.

Electromyography of the right limb's vastus medialis (VM), biceps femoris (BF), tibialis anterior (TA) and gastrocnemius medialis (GM) muscles were recorded using a Trigno wireless acquisition system (Delsys Inc., Boston, MA, USA) at a sampling rate of $1000 \mathrm{~Hz}$, according to SENIAM guidelines. Surface electrodes with an inter-electrode distance of $10 \mathrm{~mm}$, 4-bar formation, and bandwidth of 20-450 $\mathrm{Hz}$, were applied to the midline of the palpated belly muscle, parallel to the muscle fiber orientation. 
Table 1: Subject characteristic . $_{\text {. }}$

\begin{tabular}{|l|c|c|c|}
\hline & Older adults & Young adults & $p$-Value \\
\hline Age (years) & $69.9 \pm 3.8$ & $27.5 \pm 5.1$ & $<0.001$ \\
\hline Walking_speed $(\mathrm{m} / \mathrm{s})^{\text {a }}$ & $1.3 \pm 1$ & $1.9 \pm 2$ & $<.01$ \\
\hline Weight $(\mathrm{Kg})$ & $68.0 \pm 8.2$ & $68.3 \pm 17.2$ & 0.39 \\
\hline Height $(\mathrm{m})$ & $1.6 \pm 1$ & $1.7 \pm 1$ & 0.19 \\
\hline
\end{tabular}

Notes: Values are expressed as mean \pm SD and median (minimum-maximum); ${ }^{*}$ independent ttest; a Average fastwalking speed of the task

Recording points were shaved and cleaned with alcohol and then the electrodes were affixed to the skin with hypoallergenic tape. The electrodes were further secured to reduce motion artifact using flexible, non-adhesive wrap encircling the thighs (Retelast $^{\mathrm{TM}}$ Tubular Stretch Bandage Large, 3M Healthcare). Both EMG and kinematics were recorded using an infrared motion capture system (Qualisys AB, Gothenburg, Sweden). Gait events for heel strike and toe-off were computed using only kinematic data based on the heel and toe markers trajectories [12]. The EMG data were processed to extract the average linear envelope during gait cycle. EMG signals were bandpass filtered (Butterworth digital, fourth order, $20 \mathrm{~Hz}-500 \mathrm{~Hz}$ ), rectified and the RMS of the signal was obtained using $100 \mathrm{~ms}$ moving continuous windows (Visual 3D v.5, C-motion Inc., Rockville, MD, USA). RMS EMG was quantified and averaged over 30 consecutive cycles of the following phases: absorptive phase (from heel strike to peak knee flexion), and propulsive phase (from peak knee flexion to toe off). All data were normalized and expressed as a percentage of the maximal EMG detected during the first time point of the task, which corresponds to the first 30 seconds of the trial. The EMG signal were time normalized to the gait cycle starting from heel strike of the right foot to the subsequent ipsilateral heel strike. The average of 30 gait cycles provided one representative curve for each participant muscle activation data. Muscle amplitude data was ensembled averaged to obtain one curve per muscle for all participants at the five time points.

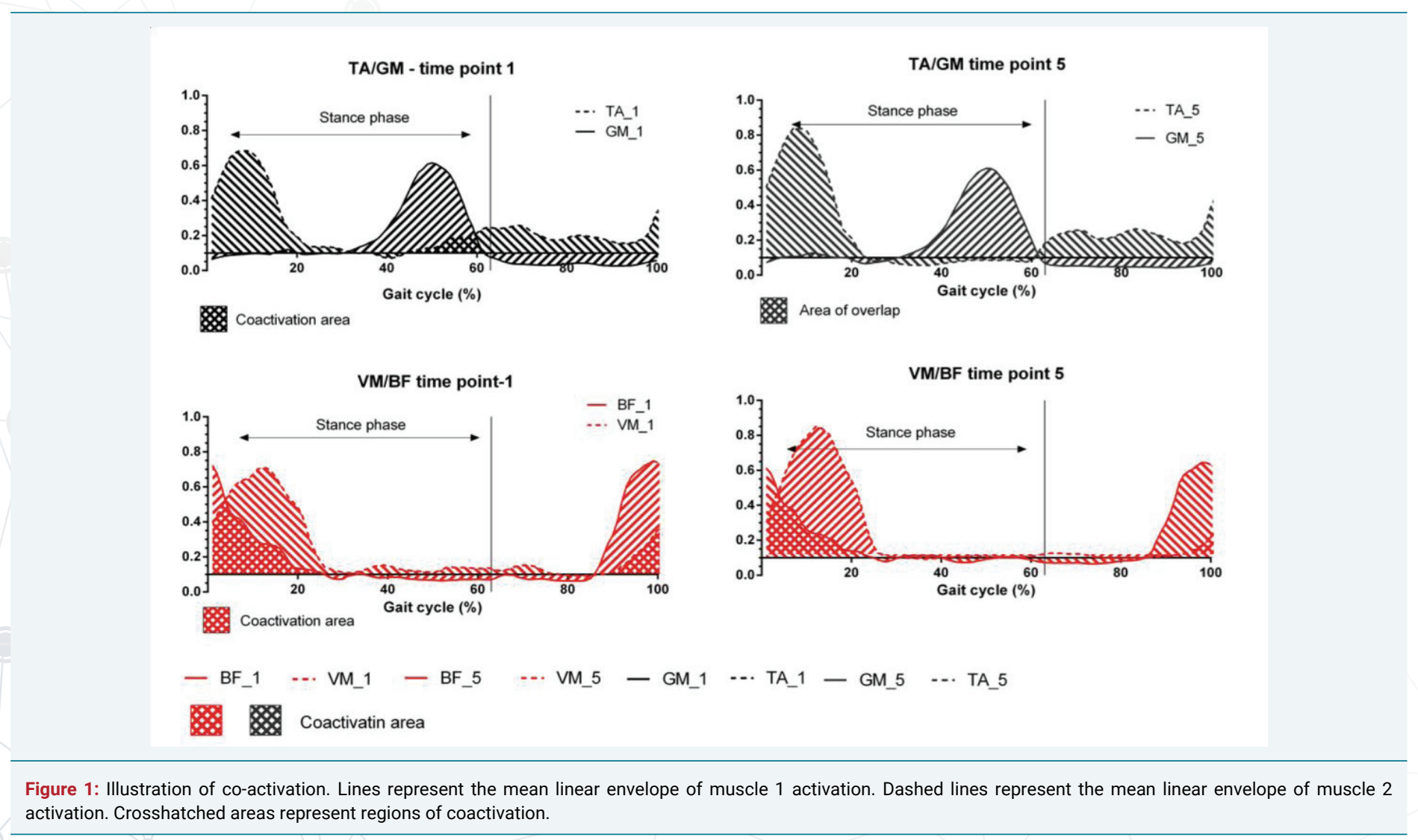


The magnitude of MG-TA and BF-VM coactivations were quantified with coactivation index (CoI) and calculated across the loading response portion of the gait, from 0 to $25 \%$ of the stance phase; the midstance and propulsive phase of support, from 25 to $100 \%$ of the stance phase; and the first and second half of the swing phase (Figure $2 \mathrm{a}$ ). The co-activation index was calculated using the following equation:

RMS EMG TA absorptive phase

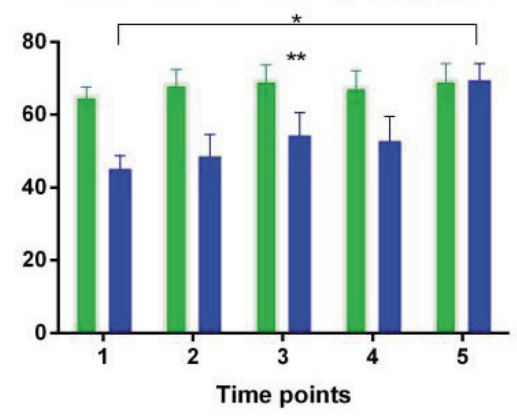

RMS EMG GM absorptive phase

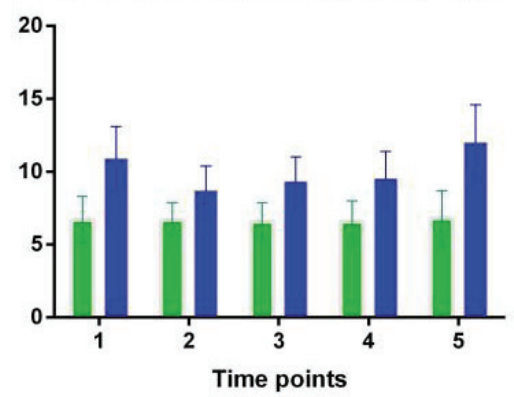

RMS EMG VM absorptive phase

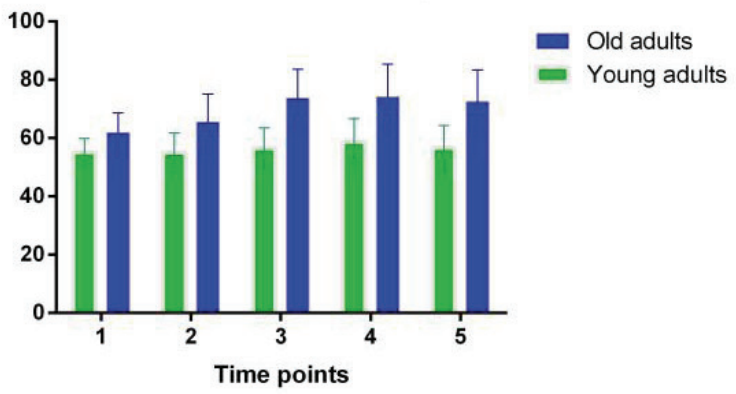

RMS EMG BF absorptive phase
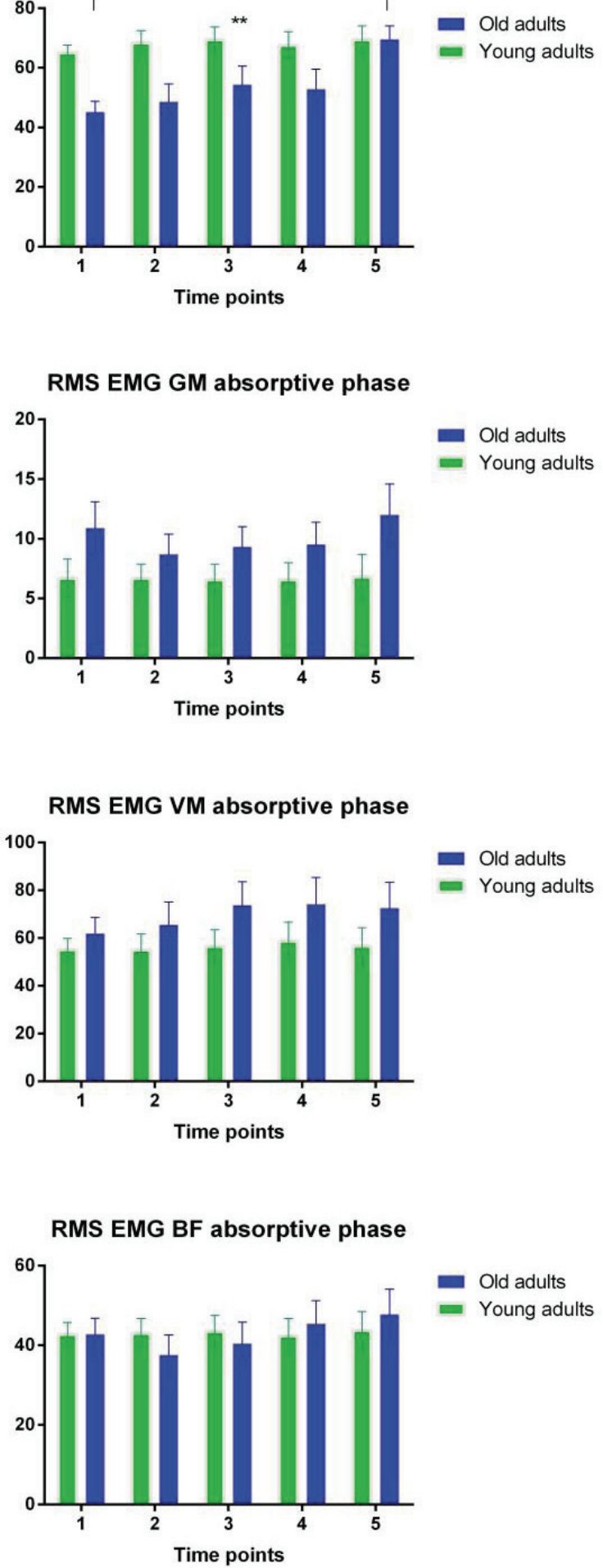

- Old adults

- Young adults
RMS EMG TA propulsive phase

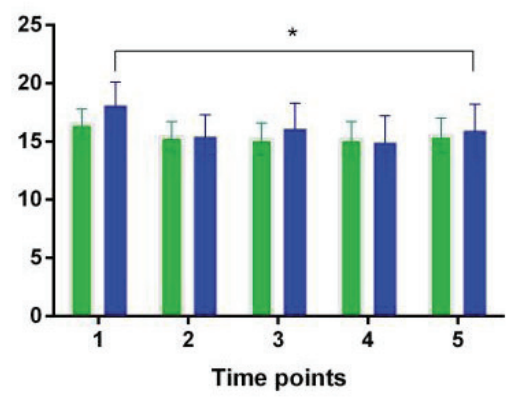

Old adults

- Young adults

RMS EMG GM propulsive phase

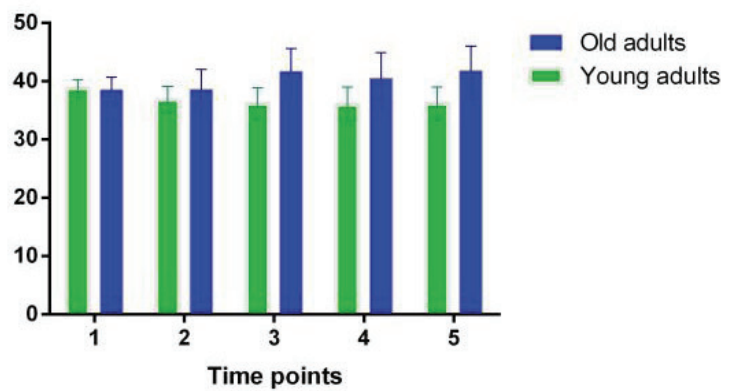

RMS EMG VM propulsive phase

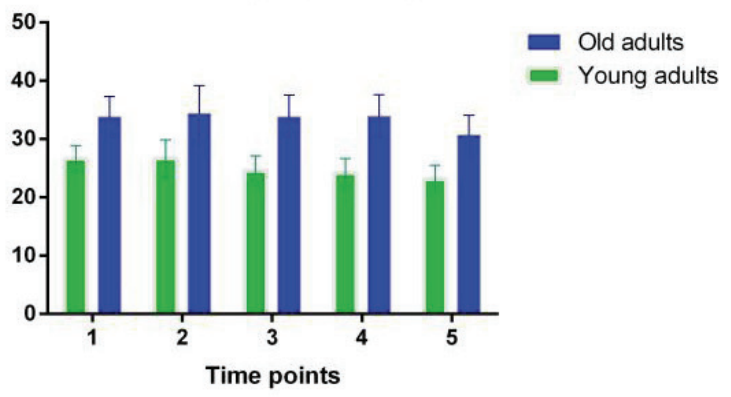

RMS EMG BF propulsive phase

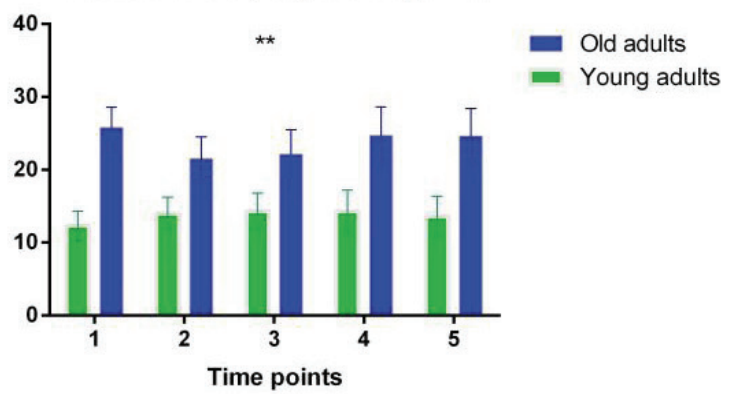

Figure 2: The changes of muscle activities in Vastus Medialis (VM), Biceps Femoris (BF), Tibialis Anterior (TA), and Gastrocnemius Medialis (MG) for adults and elderly over time. *represents significant changes over time; **represents significant differences between groups. 


$$
C I=\frac{\int E M G_{A N T}}{\int E M G_{A N T}+E M G_{A G}} \times 100 \%
$$

Where $E M G_{A N T}$ is the magnitude of EMG from the lower muscle activity, and $E M G_{A G}$ is the magnitude of EMG from the higher muscle activity [13]. Respective values of CoI were obtained from the averaged values for each participant and submitted for statistical analysis.

\section{Data analysis}

Data is reported as means \pm standard deviations. Separate mixed model repeated measures ANOVAs (2x5) were used to detect differences between age groups and within groups for time and interaction effects for all the dependent variables. All analyses were performed in SPSS (version 24, IBM SPSS, Chicago, IL.), with significance accepted at $P<0.05$.

\section{RESULTS}

Normalized RMS EMG amplitude during the first part of the stance phase (0-25\%) were not different in young adults in comparison to the older people. The assignment protocol significantly increased RMS EMG of TA in the old adults over the absorptive phase, $\mathrm{F}(1.7,31.4)=4.66, p=0.021$, with significant differences between elderly and the young subjects, $\mathrm{F}(1,18)=5.81, p=0.027$. For the rest of the support phase (25 to $100 \%$ of the stance phase), RMS activity for the TA decreased to a similar extent for both age groups, $\mathrm{F}(4,72)=4.230, p=0.004$, (Figure 2 ). Overall, the elderly demonstrated higher coactivation indexes than the younger subjects (Figure 3). There were no significant time effect CoI during the absorptive phase (Figure 3a,3e). Meanwhile, coactivation between $\mathrm{VM}$ and $\mathrm{BF}$ in the adults during the midstance and propulsive phase of support decreased significantly relative to the elderly, $\mathrm{F}(4,64)=3.164, p=0.02$, with significant difference between groups, $\mathrm{F}(1,16)=7.192, p=0.016$ (Figure 3f). Interaction effects were not significant. During the first half of the swing phase, young adults had no coactivation between the pair of muscles. The analysis was computed only for the older
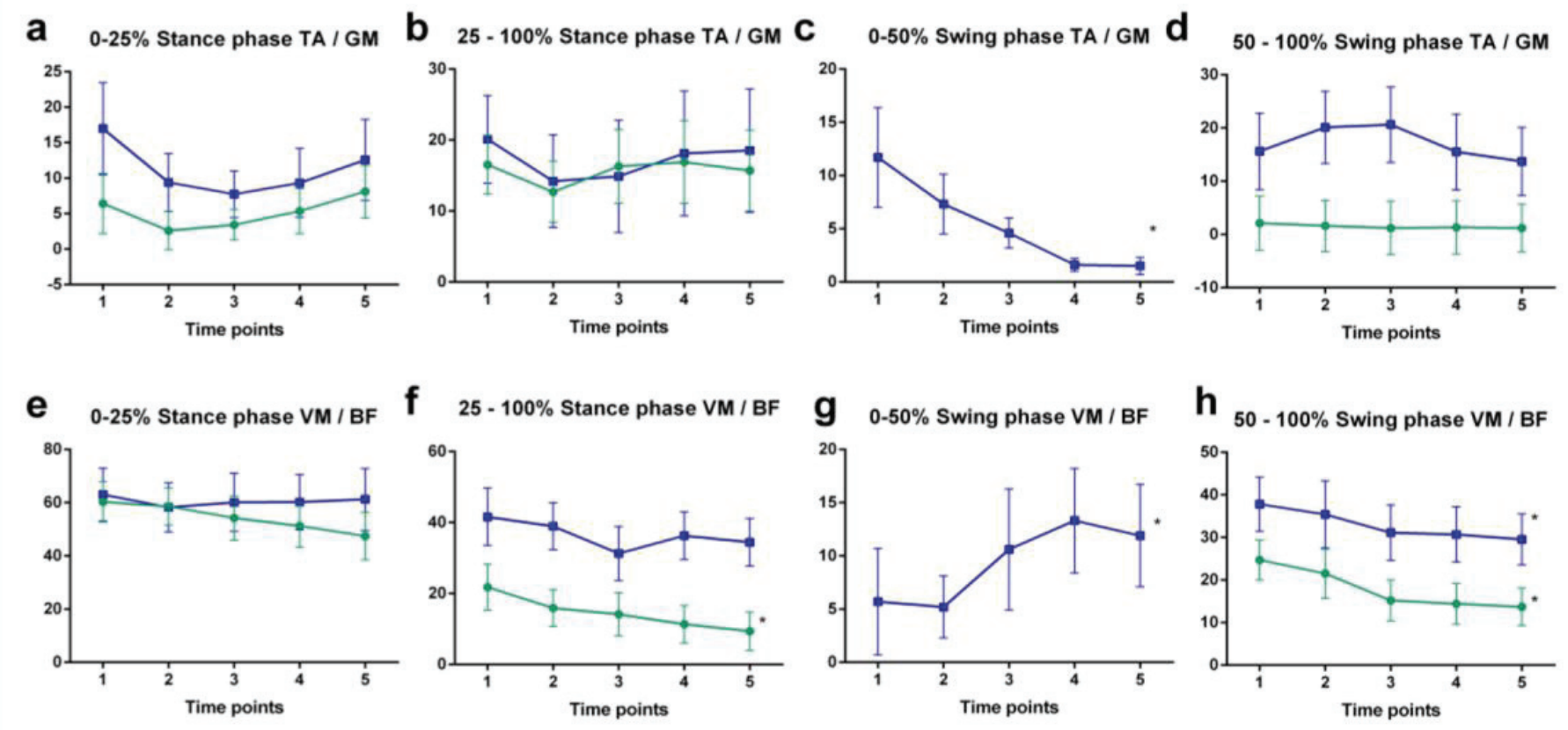

Old adults $\quad \rightarrow$ Young adults

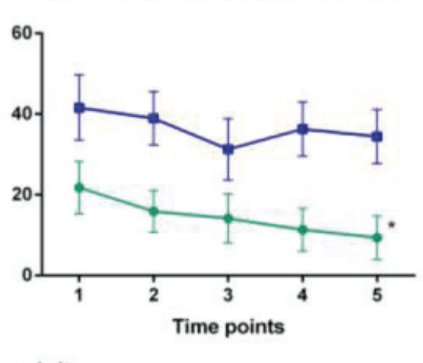

Figure 3: Changes during the activity of the coactivation indices between older (blue line) and younger adults (green line). *represents significant differences over time. 
adults. During this phase, CoI between TA and GM decreased significantly $(p=0.02)$, whilst coactivation between VM and BF increased ( $p=0.01$ ) (Figure 3c, 3g). During the second half of the swing phase, CoI between VM and BF significantly decreased in both groups, $\mathrm{F}(2.1,37)=5.274, p=0.009$, (Figure $3 \mathrm{~h}$ ).

\section{DISCUSSION}

We hypothesized that older adults would demonstrate increased muscle activation and coactivation when compared with younger adults. Overall, in the present study, we found that elderly individuals exhibited higher levels in muscle activation and coactivation about the thigh and shank than young adults, which is consistent with previous studies $[6,14,15]$. Higher muscle coactivation is expected because of the age-related reduced stretch-reflex excitability imposed by central and peripheral processes [16]. We also hypothesized that older adults would demonstrate increased muscle activity and increments in the magnitude of agonist and antagonist muscle coactivation throughout the task. In support of this hypothesis, we found significant increases in tibialis activity during the absorptive phase. However, in general, muscle coactivation decreased during fast walking in both age groups. Excepted by the significant increment in the coactivation between tibialis anterior and gastrocnemius medialis during the first half of the swing phase in old adults.

Fatigue effect on muscle coactivation is contradictory and it seems to rely on the type of task. An isotonic fatigue protocol did not alter muscle coactivation of the knee flexors or extensors [16]. However, increased ankle coactivation were detected after an isokinetic fatigue protocol [18]. Meanwhile, fatigue induced by cyclic activities, such as walking and cycling, reduced coactivation levels [7,19]. Indeed, alterations in coactivation levels in the knees and ankles exhibited during fast-walking in the present study were similar to those previously found in Nagano and collaborators [7]. Therefore, the overall reduction in muscle coactivation levels observed in the present study may have been caused by the prolonged time walking at a faster pace, and ultimately to fatigue.

Successful walking kinematics requires generating appropriate motor patterns. Muscle coactivation plays an important role in the coordination of the lower limbs in elders throughout the gait cycle [4]. Hence, we have addressed the interpretation of results regarding the role of muscle activation and coactivation within the gait cycle. The reduction of coactivation levels at the knee during terminal swing in the present study has been suggested to be a safety motor strategy [20]. At this phase of the gait, the swing leg is moving forward in preparation for load acceptance, an unexpected disturbance at this point requires a fast response from the knee. Higher levels of coactivation may affect the capacity of the knee extensors to produce force in a short period of time [21]. On the other hand, the reduction of coactivation at the knee, reported in the presentstudy, may have been the result of fatigue caused by the exertion of the activity, which may cause a delay response in producing knee joint moment. Previous studies suggest that old adults may adopt a strategy of redistributing the total available torque by using proportionally less knee torque and more hip torque [22]. In this case, further analysis should verify if fast-walking compromises hip performance, since a distal to proximal shift in joint kinetics requires greater efforts of the proximal muscle extensors.

Interestingly, most of the alterations in coactivation level were found in the swing phase. Ankle coactivation reduced significantly in the first half of the swing phase, but maintained its levels in the latter half. While knee coactivation significantly increased in the first half of the swing phase, it subsequently decreased in the second half of the swing phase. The principal swing phase task is the progression of the foot of the swing limb from the previous to the next support position [23]. Risk of falls by tripping is strictly associated to the swing toe trajectory, which is especially sensitive to the coordinated movement of the lower limb joints. In previous work Nagano and 
collaborators [9], have demonstrated the potential risk of tripping following a period of fast-walking activity. The alteration on coactivation in the ankle and knee reported herein may affect the mechanical efficiency of the swing limb, therefore affecting toe trajectory. The reduction in ankle coactivation in the first half of the swing phase throughout the task could have been caused by fatigue or yet, due to an increment in the dorsiflexor activity to ensure safety values of the toe height.

Changes in muscle activation and coactivation emerged during the task, which could ultimately affect the coordinated motion of the lower limb segments. Additionally, fatiguing of the lower extremity musculature has been suggested to influence gait variables pertinent to slip initiation and recovery phase [21]. Alterations in knee and ankle muscle activation due to fatigue were more prominent in specific sub phases of the gait, such as landing and swing phase. Our results suggest that these alterations may accommodate potential concerns with respect to risk of falls. Some limitations of this study were the absence of kinetic analysis and the small sample size. Consequently, the findings presented in this paper provide a baseline for further research and may be used in studies regarding the effect of fast-walking on muscle activation. Further studies could extend the analysis of discrete parameters to intra-stride analysis, which could provide more information regarding the fatigue effects on muscle activity within the gait cycle.

In summary, fast-walking induced neuromuscular adaptations consistent with previously reported studies based on fatigue. Most of the alterations found in the present study occurred at sub phases of gait associated to critical events that may increase risk of falls by tripping or slipping. It is therefore of interest to perform subsequent analysis regarding the biomechanical alterations that may be caused by fast-walking activity.

\section{REFERENCES}

1. Eurostat (2015) People in the EU - who are we and how do we live?

2. Tinetti ME, Speechley M. Prevention of falls among the elderly. N Engl J Med. 1989; 320: 1055-1059. Ref.: https://goo.gl/bT5qfS

3. Gandevia SC. Neural control in human muscle fatigue: Changes in muscle afferents, moto neurones and moto cortical drive. Acta Physiol Scand. 1998; 162: 275-283. Ref.: https://goo.gl/7vBovO

4. Lee LW, Kerrigan DC. Identification of Kinetic Differences Between Fallers and Nonfallers in the Elderly1. Am J Phys Med Rehabil. 1999; 78: 242-246. Ref.: https://goo.gl/251ra3

5. Hortobagyi T, DeVita P. Muscle pre- and coactivity during downward stepping are associated with leg stiffness in aging. J Electromyogr Kinesiol. 2000; 10: 117-126. Ref.: https://goo.gl/c6gx10

6. Peterson DS, Martin PE. Effects of age and walking speed on coactivation and cost of walking in healthy adults. Gait Posture. 2010; 31: 355-359. Ref.: https://goo.gl/b4TYIP

7. Pereira $\mathrm{M}$, Gonçalves $\mathrm{M}$. Effects of fatigue induced by prolonged gait when walking on the elderly Human Movement. 2011; 12: 242-247. Ref.: https://goo.gl/ZZiJwC

8. Fan Y, Li Z, Han S, Lv C, Zhang B. The influence of gait speed on the stability of walking among the elderly. Gait Posture. 2016; 47: 31-36. Ref.: https://goo.gl/lkmB42

9. Nagano H, James L, Sparrow WA, Begg RK. Effects of walking-induced fatigue on gait function and tripping risks in older adults. J Neuroeng Rehabil. 2014; 11, 155. Ref.: https://goo.gl/kOw0vX

10.Schmitz A, Silder A, Heiderscheit B, Mahoney J, Thelen DG. Differences in lower-extremity muscular activation during walking between healthy older and young adults. J Electromyogr Kinesiol. 2009; 19: 1085-1091. Ref.: https://goo.gl/CqXrSa

11.Borg GA. Psychophysical bases of perceived exertion. Med Sci Sports Exerc. 1982; 14: $377-381$ Ref.: https://goo.gl/ySQrlz

12.Zeni JA Jr, Richards JG, Higginson JS. Two simple methods for determining gait events during treadmill and overground walking using kinematic data. Gait Posture. 2008; 27: 710-714. Ref.: https://goo.gl/vHmpdq

13.Kellis E, Arabatzi F, Papadopoulos C. Muscle co-activation around the knee in drop jumping using the co-contraction index. J Electromyogr Kinesiol. 2003; 13: 229-238. Ref.: https://goo.gl/BmDBvg

14. Hortobágyi T, Solnik S, Gruber A, Rider P, Steinweg K, et al. Interaction between age and gait velocity 
in the amplitude and timing of antagonist muscle coactivation. Gait Posture. 2009; 29: 558-564. Ref.: https://goo.gl/A7IE5b

15. Mian OS, Thom JM, Ardigo LP, Narici MV, Minetti AE. Metabolic cost, mechanical work, and efficiency during walking in young and older men. Acta Physiol (Oxf). 2006; 186: 127-139. Ref.: https://goo.gl/MpOk2h

16. Obata $\mathrm{H}$, Kawashima N, Akai M, Nakazawa K, Ohtsuki T. Age-related changes of the stretch reflex excitability in human ankle muscles. J Electromyogr Kinesiol. 2010; 20: 55-60. Ref.: https://goo.gl/XWN8s0

17. Longpré HS, Potvin JR, Maly MR. Biomechanical changes at the knee after lower limb fatigue in healthy young women. Clin Biomech (Bristol, Avon). 2013; 28: 441-447. Ref.: https://goo.gl/OwOp46

18. Granacher U, Gruber M, Forderer D, Strass D, Gollhofer A. Effects of ankle fatigue on functional reflex activity during gait perturbations in young and elderly men. Gait Posture, 2010; 32: 107-112. Ref.: https://goo.gl/nRBvXS

19. Hautier CA, Arsac LM, Deghdegh K, Souquet J, Belli A, et al. Influence of fatigue on EMG/force ratio and cocontraction in cycling. Med Sci Sports Exerc. 2000; 32: 839-843. Ref.: https://goo.gl/v6rQJt

20. Pereira MP, Gonçalves M. Muscular coactivation (CA) around the knee reduces power production in elderly women. Arch Gerontol Geriatr. 2011; 52: 317-321. Ref.: https://goo.gl/ooGwxU

21. Parijat $P$, Lockhart TE. Effects of quadriceps fatigue on the biomechanics of gait and slip propensity. Gait Posture. 2008; 28: 568-573. Ref.: https://goo.gl/fOZyz5

22. Hortobágyi T, Mizelle C, Beam S, DeVita P. Old Adults Perform Activities of Daily Living Near Their Maximal Capabilities. J Gerontol A Biol Sci Med Sci. 2003; 58: M453-M460. Ref.: https://goo.gl/Lm6owH

23. Winter DA. Foot trajectory in human gait: a precise and multifactorial motor control task. Phys Ther. 1992; 72: 45-53; discussion 54-46. Ref.: https://goo.gl/8mXUr7 